\title{
An ergonomic study of work related musculoskeletal disorder risks in Indian Saw Mills
}

\author{
Qutubuddin S.M. ${ }^{1}$, S.S.Hebbal ${ }^{2}$, A.C.S.Kumar ${ }^{3}$ \\ 1,2 Department of Industrial \& Production Engineering, P.D.A. College of Engineering, Gulbarga \\ 3 JNTU College of Engineering, Hyderabad
}

\begin{abstract}
Musculoskeletal disorders (MSDs) are amongst the most common work-related problems throughout the world and India is no exception. MSD risk factors can be an appropriate base for planning and implementing ergonomics intervention programs in the workplace. The present study is focused on identifying the occupational health and safety risks of workers in Indian sawmills. The workers in saw mills are exposed to MSD risks, hard environmental conditions (high temperatures, slippery and uneven ground), heavy works (manual handling of loads, awkward postures, ) and dangerous tools and machineries such as chainsaws, band saw, chippers, etc. . In this study we have considered different logger groups working in saw mills in Karnataka State and analyzed their MSD risk exposure, occupational health hazards and noise exposure. In a developing country like India, where labor is cheap and most of the workers are economic migrants, proper occupational health, hygiene and ergonomic factors are neglected. The workers accept the adverse working conditions as part of the job and mostly work in such work environments. NORDIC musculoskeletal questionnaire was used to analyze areas of pain and their occurrence in the past. Postural analysis using REBA, RULA indicates that the workers are working above the safe limit. The average REBA score observed was 7.5. The hazardous postures and MSD's were also justified by the questionnaires and VAS (Visual Analogue Scale) techniques. Moreover the workers were exposed to noise levels above the OSHA's safe limits for prolonged time. These methods indicated that different body parts at specific postures are vulnerable to injury and musculoskeletal disorders and warrant immediate ergonomics intervention
\end{abstract}

Keywords -Ergonomics, Musculoskeletal disorders, saw mill, RULA, REBA, Noise

\section{Introduction}

Work-related musculoskeletal disorders (WMSDs) are a major concern in industry which can also compromise competitiveness due to costs related to worker compensation, labour turnover, absenteeism, poor quality and reduced productivity [1].There is a growing concern to improve productivity, safety, and quality in manufacturing industries. Some of the common problems of the small scale and unorganized sector industries are improper workplace design, ill-structured jobs, mismatch between workers abilities and job demands, adverse environment, poor human-machine system design, poor working postures and inappropriate management programs. They lead to workplace hazards, poor workers' health, mechanical equipment injuries, disabilities, Work related Musculoskeletal Disorders (WMSDs) and in turn reduce worker productivity and product/work quality and increase cost. It would, therefore, be extremely difficult to attain the objectives of the manufacturing industries without giving proper consideration to ergonomics. Effective application of ergonomics in work system design can achieve a balance between worker characteristics and task demands. This can enhance worker productivity; provide worker safety, physical and mental well-being and job satisfaction. Many research studies have shown positive effects of applying ergonomics principles in workplaces, occupational health and safety, machine design, job design, environment and facilities design.

The literature review and epidemiological studies have shown that in the genesis of the WMSD three sets of risk factors can be considered [2]

- Physical factors - e.g., sustained or awkward postures, repetition of the same movements, forceful exertions, hand-arm vibration, all-body vibration, mechanical compression, and cold;

- Psychosocial factors - e.g., work pace, autonomy, monotony, work/rest cycle, task demands, social support from colleagues and management and job uncertainty;

- Individual factors - e.g., age, gender, professional activities, sport activities, domestic activities, recreational activities, alcohol/tobacco consumption and, previous WMSD.

However, there is still a low level of acceptance and limited application of ergonomics in the manufacturing industries, especially in the small and medium industries and the unorganized sector. Neglect of ergonomic principles brings inefficiency and pain to the workforce. An ergonomically deficient workplace can cause physical and emotional stress, low productivity and poor quality of work. Assessment of exposure levels to MSD risk factors can be an appropriate base for planning and implementing interventional ergonomics programs in the workplace. The objective of this study is to analyze the working postures of workers engaged 
in various activities carried out in a saw mill, by applying different postural analysis tools, and to identify the various risk factors associated with MSDs.

\subsection{Saw Mills in India}

The majority of saw mills in India are small scale and unorganized. As an unorganized sector, no statistical data are available as to the number of people employed, accident rates, working conditions, and other problems. The nature of the work done by workers in these saw and the types of mills and the equipment and materials they handle present many on-the-job hazards. These hazards and injuries resulting from such incidence include: being caught-in or struck by machinery, falling from a height, ergonomic hazards resulting from lifting of heavy loads, reaching for objects, repetitive work, and poor work posture, twisting or reaching, and breathing in fine particles of saw dust while working for increase productivity. Besides the hazards inherent in this profession are the unfavorable weather conditions and noise pollution that is injurious to human health. [3] Workers are also prone to injury when removing scrap or finished pieces from the table, kickback injuries as a result of incorrect blade height etc. As a result of the high level of human (manual handling) involvements in sawmilling operations, workers are exposed to higher levels of risks associated with log handling and machine operation, environmental hazards, work related body injuries, and in extreme cases, death.[4] In order to fully assess the safety environment in the mill sites within these areas, an overview of the wood processing industry activities and associated hazards are necessary.

The study was carried out in Saw mills situated in northern Karnataka in India where thousands of workers are engaged in these activities. As it is an unorganized sector, no statistical data are available to date as to the accident rates occurred, the number of people employed, and other problems. Hence the only means of these data are through conversations and direct dialogue with the people associated with this profession. Saw milling involves more work than it appears because most of the work is carried out manually. Saw workers, especially sawyers, are exposed to high concentrations of saw dust while cutting the wood. The incidences of Work Related Musculoskeletal Disorders (WMSDs) are quite common in this sector. Many literature shows the prevalence of WRMSD's in such unorganized sectors like brickmaking, oilmills, stonecrushing, handicrafts, leather etc. but little work has been done on saw mills. Saw-filer position was chosen for assessment and the study gave the high rate of upper extremity MSIs observed during the period reviewed visits.[5] But a lack of study on the postural analysis of the saw mill workers was observed in this region. The workers are subjected to the high noise of the horizontal and vertical band saws for durations above the safe limit. The workers also carry heavy loads on a regular basis causing health problems, apart from other improper work postures. The workers are assigned work on a daily wages basis. Thus it appears that to date there is dearth of data in the Indian scenario about the different risk factors associated with saw mill. Since it's a cluster industry in this region and employing thousands of workers, it is necessary to analyze the sector and to identify the hazards and the level of risk implicit in performing the required tasks. This study is an attempt in that direction.

\section{Materials And Methods}

The study was carried out at saw mills situated in northern Karnataka. A video of different work activities involved in saw mill like log moving, loading in band saw machine, cutting, blade sharpening, chain saw, showing the different movements and body postures during an activity was recorded. Later the video was cropped after every 10 seconds to get snapshots of the posture of the worker for analysis. Snapshots of about 110 workers working in different activities were selected randomly. The snapshots were used for the scoring of REBA and RULA sheets.

\subsection{Questionnaire and interview technique}

As a first step of the study a questionnaire was administered which comprised questions pertaining to different problems related to a particular task such as: normal daily activity; discomfort in the different body parts; working hours; resting periods; and total working duration. NIOSH discomfort survey was used for mapping the different areas of pain with its intensity in the body along with the factors like duration and frequency. Also NORDIC musculoskeletal questionnaire was used to analyze areas of pain and their occurrence in the past.

\subsection{Visual Analogue Scale}

The Visual Analog or Analogue Scale (VAS) [6] is designed to present to the respondent a rating scale with minimum constraints. Respondents mark the location on the $10-\mathrm{cm}$ line corresponding to the amount of pain they experienced. This gives them the greatest freedom to choose their pain's exact intensity. It also gives the maximum opportunity for each respondent to express a personal response style. The VAS frame measures exactly $10 \mathrm{~cm}$. The distances from zero to the markings in $\mathrm{cm}$ are result indicators to be processed as continuous variables for statistical analysis. 


\subsection{Postural analysis}

Different techniques were applied for postural analysis of work related musculoskeletal risk factors. These methods were Rapid Entire Body Assessment (REBA) method . REBA (Rapid Entire Body Assessment) was developed by Hignett, S. and McAtamney, [7] to provide a quick and easy observational postural analysis tool for whole body activities (static and dynamic giving musculoskeletal risk action level. The development of REBA is aimed to divide the body into segments to be coded individually with reference to movement planes. It provides a scoring system for muscle activity caused by static, dynamic, rapid changing or unstable postures. It reflects that coupling is important in handling of the loads but may not always be via the hands. It also gives an action level with an indication of urgency. This method was specifically developed to be useful for assessing MSD risks/working postures found in healthcare and other service industries. However, it can be used to assess a variety of tasks, in any setting, where: the whole body is being used, the posture is static, dynamic, rapidly changing, or unstable, or animate or inanimate loads are being handled either frequently or infrequently. The scoring sheet for calculating REBA score is shown in fig.1.

Rapid Upper Limb Assessment Method (RULA) method . RULA is a quick survey method for use in ergonomic investigations of workplaces where MSD's are reported. It is a screening tool that assesses biomechanical and postural loading on the body. It focuses on the neck, trunk and upper limbs, and is ideal for sedentary workers.[8]. It is a simple, quick and easy to complete. RULA scores indicate the level of intervention required to reduce MSD risks. It compliments other ergonomic methods. RULA scoring sheet is shown in fig.2. RULA assesses the posture, force and movement associated with sedentary tasks such tasks include computer tasks, manufacturing or retail tasks where the worker is seated or standing without moving about. This tool requires no special equipment in providing a quick assessment of postures of the neck, trunk and upper limbs along with muscle function and the external loads experienced by the body. A coding system is used to generate an action list which indicates the level of intervention required to reduce the risks of injury due to physical loading on the operator.

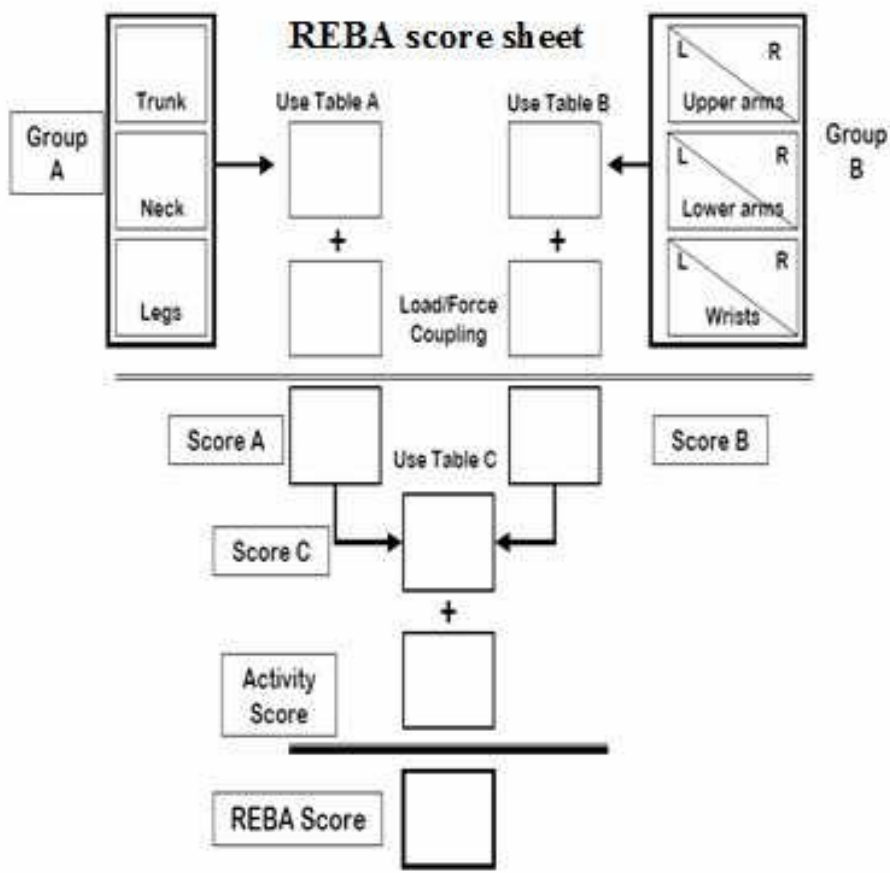

\begin{tabular}{cccc}
\hline Action level & REBA Score & Risk level & Action \\
\hline 0 & 1 & Negligible & Not necessary \\
& & & \\
1 & $2-3$ & Low & May be necessary \\
2 & $4-7$ & Medium & Necessary \\
3 & $8-10$ & High & Necessary soon \\
4 & $11-15$ & Very high & Necessary now \\
\hline
\end{tabular}

Figure 1. REBA score sheet 


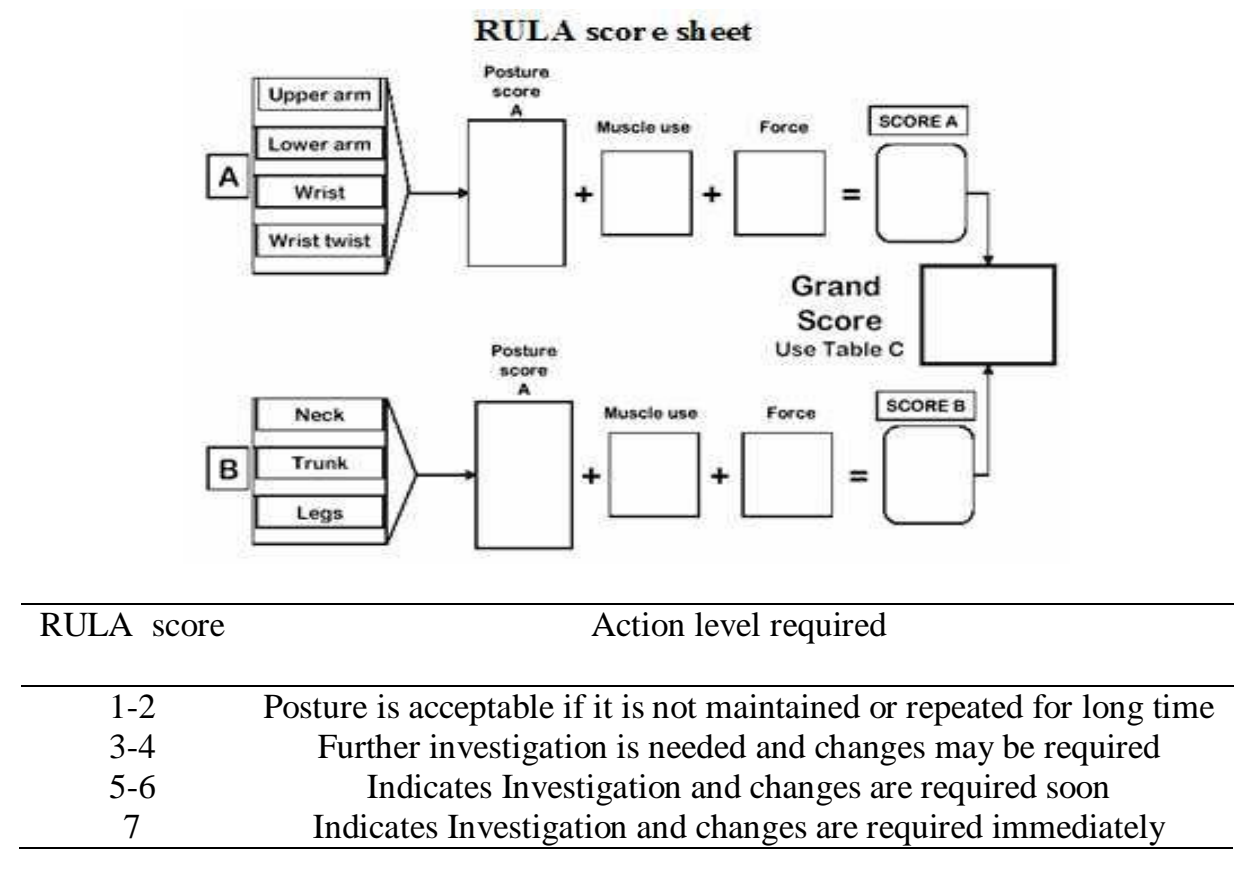

Figure 2. RULA score sheet

\subsection{Noise Assessment}

Noise is an unwanted or damaging sound that may damage the hearing and cause other health effects such as stress, hypersensitivity to noise, increased blood pressure and increased heart rate. It can also interfere with communication at work, which could lead to accidents. Noise-induced hearing loss is one of the most common occupational injuries, resulting in health problems for many workers and it presents a significant social and economic cost. Noise has a major effect on one's performance at work, since a peaceful environment may result in good mood and hence in good output from the worker whereas lot of noises over long time may cause hindrance in the work output of the worker.[9] .The wood working machinery like Band saws, are widely used in the wood industry. Without any measures to reduce noise at source, they can produce noise levels of over 90 $\mathrm{dB}$ (typically $100 \mathrm{~dB}$ at the operator position). At this level of noise, an employee's daily personal noise exposure is too high well above the OSHA's set limits of $90 \mathrm{~dB}$ at operator's level for $8 \mathrm{hrs}$ as inferred from this study.

\subsection{Direct observation and activity analysis}

\section{Results And Discussion}

The typical south Indian saw mill comprises of a manager in charge of the plant, assisted by the plant superintendent saw mill foreman, master mechanic, bookkeeper, and shipping clerk. Most work at the saw mill is done manually without much use of powered vehicles. In nearly 70 sawmills in this region all movement of the logs from unloading section to the carriage, from carriage to the delivery point is done manually, many such manual activities were observed for few minutes to 2 hours at a stretch for both seated and standing position of the workers. The work involved predominant effort from the trunk and arms. The work in a typical saw mill starts right from the unloading of raw wooden logs (up to 5 quintals) into the unloading section of the mill. Then these logs are moved to the horizontal band saw with the help of levers as shown in figs. 3.
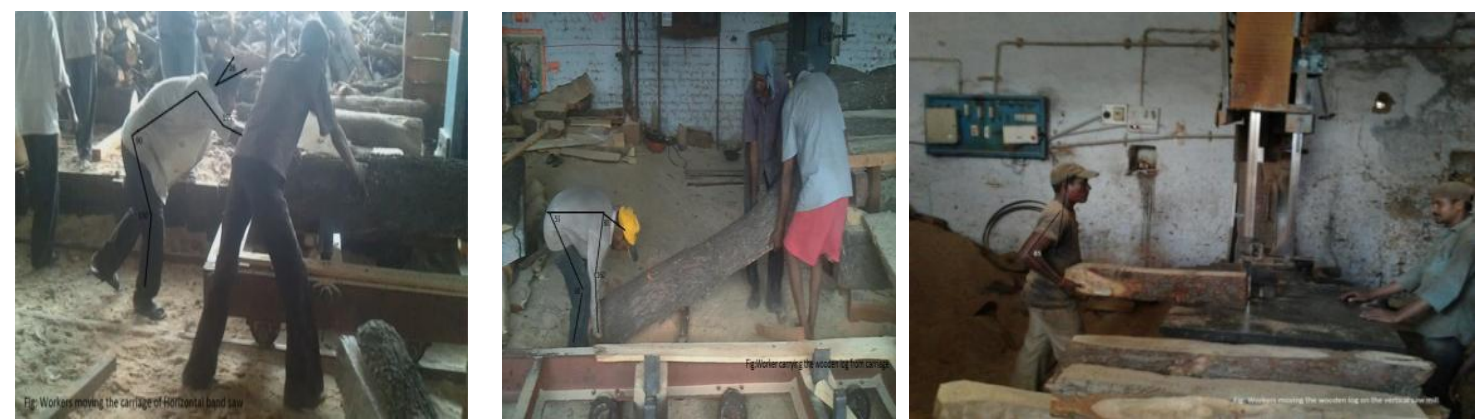

Figure 3: diferent postures of workers in saw mill 
NIOSH's discomfort survey was used for mapping the different areas of pain, the results also showed that the discomfort frequency among the workers was frequently i.e. once a week for almost $65 \%$ of the workers, and discomfort intensity of severe among 33\% workers and moderate among $47 \%$ and mild among the $20 \%$ workers, the duration of intensity was observed to be within 1 to $24 \mathrm{hrs}$ among $75-80 \%$ of the workers. Also NORDIC questionnaire suggested that pain was common among the workers of saw mills and was being experienced frequently during last 1 year. This indicates the problem of MSDs is serious in saw mills and must be attended very soon.

Table 1: Worker Demographic Data

\begin{tabular}{cc}
\hline Variables & Mean $(\mathrm{SD})$ \\
\hline Age $(\mathrm{yr})$ & $37.4( \pm 9.5)$ \\
Height $(\mathrm{cm})$ & $165.2( \pm 11.3)$ \\
Weight $(\mathrm{kg})$ & $57.9( \pm 9.7)$ \\
Years of Experience & $14.7( \pm 8.6)$ \\
Duration of work per day (hours) & $9.5( \pm 1.8)$ \\
Duration of rest per day (hours) & $1.5( \pm 0.5)$ \\
Number of work days & 6 \\
Per week & \\
\hline
\end{tabular}

\subsection{Visual Analogue scale}

Respondents were asked to mark the location on the $10-\mathrm{cm}$ line corresponding to the amount of pain they experienced. This gave them the greatest freedom to choose their pain's exact intensity. It also gave maximum opportunity for each respondent to express a personal response style. Table 2 shows the Visual analogue scale ratings for a sample of 25 workers and a mean score of 6.54 indicating a high intensity of pain

Table 2: VAS rating among saw mill workers

Study groups VAS Rating

Saw mill workers $\quad 6.54 \pm 1.28$

\subsection{Noise Assessment}

Noise levels generated by sawmill saws in operation have been reported to vary from $80 \mathrm{~dB}(\mathrm{~A})$ up to $120 \mathrm{~dB}(\mathrm{~A})$. Not only cutting noise is very high, there is also the additional factor that, even when idling, saws can produce noise levels up to $95 \mathrm{~dB}(\mathrm{~A})$. It is well recognized fact in industry that noise is a serious problem with saws. One of the unique features of the noise associated with wood working machines is its intermittent nature. The noise exposure level at which precautionary actions are required to prevent hearing loss. OSHA defines the Action Level as an 8-hour time-weighted average noise exposure of $90 \mathrm{dBA}$. According to OSHA regulations, noise exposures at or above this Action Level require follow-up measures that include noise monitoring, annual audiometric testing for exposed employees, hearing protection, training and recordkeeping. The observations on the saw mill showed that the workers were exposed to sounds of 90-100 dB for more than 6 hours daily. It was observed that the workers were used to such noise levels and were not provided with any hearing protection like ear plugs.

Table 3: Noise levels of different machines

\begin{tabular}{ccc}
\hline Equipment / Machine & \multicolumn{2}{c}{ Noise level dB (A) } \\
\cline { 2 - 3 } & Min & Max \\
\hline Horizontal Bandsaw & 91.8 & 94.0 \\
Vertical Bandsaw & 94.7 & 96.4 \\
Grinder & 97.5 & 100.4 \\
Chainsaw & 87 & 91 \\
\hline
\end{tabular}

\subsection{Postural Analysis}

The working postures of the workers performing different activities were analysed using the tools RULA and REBA. The distribution of RULA score is shown in Table 4. The analysis of scores shows that $5.45 \%$ of workers are at negligible risk and $29.10 \%$ workers are at low risk where further investigation may be 
needed. The scores reveal that a majority of workers in saw mills are at medium to high risk, as shown where $42.72 \%$ workers are at medium risk and $22.73 \%$ workers are at high risk. The postures of the workers at high risk has to be changed immediately. Most of the workers in high risk category are working on vertical bandsaw machines. A necessary change was suggested for the workers in these machines.

The results of REBA assessment are shown in Table 5. According to this technique of postural analysis $7.27 \%$ of workers are working in postures which can be ignored. About $10.91 \%$ of workers are at low risk where a change in posture may be necessary. Medium risk postures account for $26.36 \%$ of workers. These are the workers working in lighter tasks like movement, holding the wooden logs, adjusting the machines. $34.54 \%$ of workers postures are at high risk where a change is required soon. The workers were suggested about the possible changes to be made. Nearly $20.92 \%$ of workers are at very high risk which requires immediate change in postures. The workers in this category are those working on Horizontal bandsaw machine, moving log by bending, twisting the body for a considerable time. The results of both RULA and REBA indicate a high percentage of workers are at high to very high risks. As compared to RULA which gives $22.73 \%$ of workers postures as high risk requiring immediate action to be taken, REBA categorizes $20.92 \%$ of workers postures under very high risk. Further the results of RULA and REBA indicate that the workers in saw mills are exposed to high risks and ergonomic interventions are necessary.

Table 4 : Distribution of RULA Score

\begin{tabular}{ccccc}
\hline $\begin{array}{c}\text { RULA } \\
\text { Score }\end{array}$ & Risk level & Action & $\begin{array}{c}\text { No.of } \\
\text { Workers }\end{array}$ & $\begin{array}{c}\text { \% age of } \\
\text { workers }\end{array}$ \\
\hline $1-2$ & Negligible & Acceptable & 6 & 05.45 \\
$3-4$ & Low & Further investigation and changes may be & 32 & 29.10 \\
needed & $\begin{array}{c}\text { Investigation and changes required soon } \\
\text { Investigation and changes required } \\
\text { immediately } \\
7\end{array}$ & Medium & 47 & 42.72 \\
& High & 25 & 22.73 \\
& & Total & 110 & \\
\hline
\end{tabular}

Table 5: Distribution of REBA Score

\begin{tabular}{ccccc}
\hline $\begin{array}{c}\text { REBA } \\
\text { Score }\end{array}$ & Risk & Action & No. of workers & \%age of workers \\
\hline & level & Not necessary & 8 & \\
$2-3$ & None & May be necessary & 12 & 07.27 \\
$4-7$ & Medium & Necessary & 29 & 26.91 \\
$8-10$ & High & Necessary and soon & 38 & 34.54 \\
$11-15$ & Very & Necessary urgent & 23 & 20.92 \\
& high & Total & 110 & \\
& & &
\end{tabular}

\section{Conclusion}

It was observed from the study and findings that in the small scale industries and the unorganized sector there is very little awareness about ergonomics, good work environment, good postures etc. Musculoskeletal disorders are present in the various activities carried out in saw mills where a significant number of workers are working in bad postures and it shows that there is a need to change the body postures. On the basis of analysis of results and scores obtained by the various tools applied in analysis of the working conditions and postures, it can be concluded that, there is a lack of ergonomics planning and methods in small scale and unorganized saw mills which are very large in numbers and employ thousands of workers. Thus the workers are under moderate to high risk of Work Related Musculoskeletal disorders (WRMSDs). The average scores of both the RULA and REBA are high and percentage of scores for neck and trunk are high for the subjects analyzed, and this evaluation shows that there is a need of investigation and immediate changes are needed in the workplace. Most operations carried out in the mills are largely supported by manual handling which often results in overexertion and a long term health hazards. 
Personal Protection devices such as earmuff or plug, hand gloves are not used adequately and are often considered as irrelevant and disturbing. Dust and wood wastes are not properly disposed. This was evident from heaps of wood shavings and saw dust accumulation around saw milling machines. Training is also important: to train and to inform operators about the correct positions and the manual movement techniques may reduce many incorrect postures The noise produced in the both the saw mills is above the desired limits and it should be reduced. Finally it was observed that none of the companies had any safety policies, materials, etc., in place, but a work methodology of most economical ways was followed irrespective of its effect on the workers. Focus should be on these challenges in practical terms to form a safety legislation, comprehensive systems approach and monitoring group in the industry to guide the management in the implementation in order to reduce or eliminate workplace hazards.

The present study recommends that there is dire need of implementation of ergonomics interventions with proper awareness among workers. In developing countries like India, the scale of use of human resources in small- medium scale in labour-intensive industries is enormous. In this situation, it must be obvious that very small improvements in working conditions, implements, tool design or working methods can lead to large benefits. It is believed that occupational health programs should focus more on the informal sector, which employs a large proportion of workers. Paying attention to occupational health and safety in this sector and improving working conditions will undoubtedly have considerable impact on the national economy and the quality of people's life.

\section{Acknowledgement}

The authors express sincere gratitude to the managements of sawmills and the workers for their cooperation and help during the completion of this study.

\section{References}

[1] Andersson ER, Economic evaluation of ergonomic solutions: part I-guidelines for the practitioner, International Journal of Industrial Ergonomics. 1992, (10),161-71.

[2] Nunes, I. L., FAST ERGO_X - a tool for ergonomic auditing and work-related musculoskeletal disorders prevention, WORK: A Journal of Prevention, Assessment, \& Rehabilitation, Vol. 34(2) ,2009, 133-148

[3] Judd H. Michael and Janice K. Wiedenbeck, Safety in the wood products industry. Forest Products Journal, Vol. 54, No. 10, 2004

[4] Segun R. Bello and Yahaya Mijinyawa., Assessment of Injuries in Small Scale Sawmill Industry of South Western Nigeriall. Agricultural Engineering International: the CIGR Journal of Scientific Research and Development. Manuscript 1558. Vol. XII, March, 2010.

[5] Troy Jones Shrawan Kumar-, Comparison of Ergonomic Risk Assessment Output in Four Sawmill Jobs, International Journal of Occupational Safety and Ergonomics (JOSE) 2010, Vol. 16, No. 1, 105-111

[6] Thomee R, Grimby G, Wright BD, Linacre JM, Rasch analysis of Visual Analog Scale measurements before and after treatment of patellofemoral pain syndrome in women, Scand J Rehabil Med 1995, 27, 145-51.

[7] Sue Hignett,Lynn McAtamney - Rapid Entire Body Assessment (REBA), Applied Ergonomics 31 (2000) $201-205$

[8] McAtamney, L. and Corlett, E. N., RULA: a survey method for the investigation of work related upper limb disorders. Applied Ergonomics, 24, 1993, 91-99.

[9] Qutubuddin S.M., Hebbal S.S. and A.C.S. Kumar, A Review on Effect of Industrial Noise on the Performance of Worker and Productivity, International Review of Applied Engineering Research. Volume 2, Number 1 (2012), pp. 43-54 